\title{
Estimating population genetic parameters and comparing model goodness-of-fit using DNA sequences with error
}

\author{
Xiaoming Liu, ${ }^{1}$ Yun-Xin Fu, Taylor J. Maxwell, and Eric Boerwinkle \\ Human Genetics Center, School of Public Health, The University of Texas Health Science Center at Houston, \\ Houston, Texas 77030, USA
}

\begin{abstract}
It is known that sequencing error can bias estimation of evolutionary or population genetic parameters. This problem is more prominent in deep resequencing studies because of their large sample size $n$, and a higher probability of error at each nucleotide site. We propose a new method based on the composite likelihood of the observed SNP configurations to infer population mutation rate $\theta=4 N_{e} \mu$, population exponential growth rate $R$, and error rate $\varepsilon$, simultaneously. Using simulation, we show the combined effects of the parameters, $\theta, n, \varepsilon$, and $R$ on the accuracy of parameter estimation. We compared our maximum composite likelihood estimator (MCLE) of $\theta$ with other $\theta$ estimators that take into account the error. The results show the MCLE performs well when the sample size is large or the error rate is high. Using parametric bootstrap, composite likelihood can also be used as a statistic for testing the model goodness-of-fit of the observed DNA sequences. The MCLE method is applied to sequence data on the ANGPTL4 gene in 1832 African American and 1045 European American individuals.
\end{abstract}

[Supplemental material is available online at http://www.genome.org. ]ava programs for calculating MCLE of $\theta$, $R$, and $\varepsilon$ are freely available at http://sites.google.com/site/jpopgen/.]

Population parameter inference is one of the most important tasks in theoretical and applied population genetics. Among all parameters, the scaled population mutation rate $\theta$ is probably the most important for modeling the evolution of a DNA locus in a population. The quantity $\theta$ equals $4 N_{e} \mu$ for diploids or $2 N_{e} \mu$ for haploids, where $N_{e}$ is the effective population size and $\mu$ is the mutation rate per generation of the locus. The mutation rate describes the basic variability of DNA sequences in a population, and many summary statistics of DNA sequences are related to $\theta$. Therefore, estimating $\theta$ plays a central role in understanding the evolution of a population. Other important population parameters include, but not limited to, the population exponential growth rate $R$, the scaled recombination rate $\rho$, and the migration rate.

One challenge of estimating these population parameters is how to incorporate sequencing error when it is not negligible, e.g., when the error rate is high and/or sample size is large. It is easy to understand that errors can bias the estimation of evolutionary or population genetic parameters with sequence samples (Clark and Whittam 1992; Johnson and Slatkin 2008). The sample size has a large impact because sequencing error increases linearly with sample size, while the expected number of true mutations increases more slowly, as implied by coalescent theory. As a rule of thumb, population genetic estimates that are uncorrected will be biased significantly if $n \varepsilon \geq \theta / L$, where $n$ is sample size (i.e., number of sequences sampled), $\varepsilon$ is the average error rate per site, and $L$ is the sequence length of the given locus (Johnson and Slatkin 2008).

The newer parallel DNA sequencing technologies have higher error rates compared to traditional Sanger sequencing, and at the same time their low cost per base pair (bp) encourages researchers

\footnotetext{
${ }^{1}$ Corresponding author.

E-mail Xiaoming.Liu@uth.tmc.edu; fax (713) 500-0900.

Article published online before print. Article and publication date are at http://www.genome.org/cgi/doi/10.1101/gr.097543.109. Freely available
} online through the Genome Research Open Access option. to conduct larger-scale sequencing projects with larger sample sizes (Shendure and Ji 2008). While a typically finished sequence produced by Sanger sequencing has an error rate of $10^{-5}$ (Zwick 2005), the error rates of the newer parallel sequencers are typically 10 -fold higher (Shendure and Ji 2008). Although technological advance may further decrease their error rate, the trend of balancing sequencing quality and quantity for the newer parallel sequencers is likely shifting toward larger quantities with higher error rates, e.g., on the scale of $10^{-4}$ on consensus reads. For some sequencing projects, such as metagenomic shotgun sequencing, each sequence can be considered as a random sample from a sequence pool of a whole population (or populations) (Whitaker and Banfield 2006), so that it is almost impossible to reduce error rate by multiple reads from the same sequence. In such cases, the error rate in the sequences can be higher than $10^{-3}$ (Shendure and Ji 2008).

In response to these challenges, new unbiased estimators for $\theta$ and other population parameters for sequence samples with error are proposed. Targeting metagenomic sequencing data, Johnson and Slatkin (2006) were probably the first to directly address the error problem. Incorporating sequencing errors via Phred quality scores, they proposed a maximum composite likelihood estimator (MCLE) for $\theta$ and $R$ using the SNP frequency spectrum. In a later study they corrected the sequencing error bias of two widely used $\theta$ estimators, Tajima's $\hat{\theta}_{\pi}$ (Tajima 1983) and Watterson's $\hat{\theta}_{S}$ (Watterson 1975), assuming known $\varepsilon$ (Johnson and Slatkin 2008). Another corrected $\hat{\theta}_{S}$ assuming known $\varepsilon$ was proposed by Hellmann et al. (2008), which is similar to Johnson and Slatkin (2008), but accounts for the uncertainty of chromosome sampling in a shotgun resequencing of pooled diploid individuals. All the above estimators of $\theta$ assume that $\varepsilon$ is known. For sequence data with unknown error rate, several estimators have recently been developed. Achaz (2008) proposed two computationally efficient, moment-based estimators. Assuming a low but unknown $\varepsilon$, the estimators simply use the SNP number and frequency spectrum, while ignoring 
singletons, on which sequencing error is most skewed. Knudsen and Miyamoto (2007) developed a full-likelihood coalescent model that incorporates missing data, sequencing error, and multiple reads of the same sequence. Both $\theta$ and $\varepsilon$ can be estimated jointly with their method. However, the computational intensity of calculating the likelihood is so high that only small sample sizes (i.e., less than 20 sequences) can realistically be considered. By ignoring singletons, Knudsen and Miyamoto (2009) modified their original algorithm to improve its accuracy and computational speed. Targeting high-coverage shotgun resequencing of a single diploid individual, Lynch (2008) proposed several methods to correct $\hat{\theta}_{\pi}$, assuming a known or unknown $\varepsilon$. Lynch (2009) further extended the method to estimate allele frequencies of each nucleotide site with multiple individuals. The combination of Lynch (2008) and Lynch (2009) provides a method to estimate $\hat{\theta}_{\pi}$ with high-coverage resequencing data of multiple individuals. Jiang et al. (2009) developed a method to estimate $\theta$ and $\rho$ for shotgun resequencing data. Although they did not incorporate error rate into their method, they suggested some refinements to make their method more robust to errors.

Recently, Liu et al. (2009) modified Fu's (Fu 1994) best linear unbiased estimator (BLUE) and proposed two types of $\theta$ estimators, based on generalized least squares (GLS) with either known or unknown $\varepsilon$. One type uses the full spectrum of SNP frequency and incorporates $\varepsilon$ into the calculation, while the other type simply ignores singletons and treats $\varepsilon=0$ for other SNP frequency classes. The two estimators either strictly or relaxedly assume that there is, at most, one error on any given nucleotide site of the whole sample, therefore their efficiency decreases with an increase of $\varepsilon$ and they are more suitable for data with $\varepsilon<10^{-4}$ and moderate sample sizes $\left(10^{2}\right.$ sequences). Another disadvantage is that they cannot handle missing data directly. For large resequencing projects and metagenomic sequencing projects, the missing data rate of the resulting sequences can be high. Especially with metagenomic shotgun resequencing, the nature of uneven coverage on different nucleotide sites will produce very different sample sizes for different sites, which can be considered as a type of missing data.

In this study we propose a MCLE of $\theta, R$, and $\varepsilon$ that is suitable for data with high error rates, large sample sizes and/or a high missing data rate. While the full likelihood method calculates the likelihood of sequences as a whole, the composite likelihood method first calculates the likelihood of each observed nucleotide site (or pair of sites) and then calculates the total likelihood of the sequences by multiplying these individual likelihoods as if they are independent (e.g., Nielsen 2000; Hudson 2001). The composite likelihood is an approximation of the full likelihood and MCLE should have larger variance than a full likelihood estimator. However, the computational burden is substantially reduced, so that in many cases a MCLE can be a good compromise between estimation efficiency and computational speed. A MCLE of $\theta$ and $R$ was proposed by Johnson and Slatkin (2006) for metagenomic sequencing data. Compared to their method, ours is more suitable for resequencing data with a large sample size because of two major differences. First, we do not assume each nucleotide read has an associated quality score, but instead use an error rate $\varepsilon$ for each nucleotide site for all samples. One reason for this is that some SNP calling software do not provide a quality score. Second, our method systematically handles cases when there are more than two allele types at a SNP site (due to sequencing error), which becomes more common when the sample size is large.

Besides parameter estimation, whether the observed sequence pattern of a gene or a DNA region fits a particular population genetic model, and which model fits the data better, are also important questions in population genetics. The composite likelihood of a DNA region can also be used as a summary statistic of the model's goodness-of-fit to the data. Therefore, the model goodness-of-fit test and model comparison can be conducted by parameter estimation followed by parametric bootstrap (see Methods for details).

Below we show the performance of our MCLEs of $\theta$ and $R$ using computer simulation, and compare our MCLE of $\theta$ with other $\theta$ estimators. Then, we demonstrate its application for both parameter estimation and the model goodness-of-fit comparison using data from a resequencing project of the ANGPTL4 gene in 1832 African Americans and 1045 European Americans (Romeo et al. 2007).

\section{Results}

\section{Simulation validation}

Coalescent simulation (see Methods) was used to investigate the properties of our MCLE and its performance with changing population parameters, including $\varepsilon, n, \theta$, and $R$. One obvious problem of MCLE is the treatment of each nucleotide site independently. Although recombination may validate the assumption for genetically unlinked sites, for any closely linked sequence the assumption is obviously invalid. To investigate the robustness of MCLE, we simulated sequences with no recombination. With different combinations of parameters, at least 10,000 samples were simulated assuming an exponential growth population model $(R>0)$ and at most three sequencing errors on any site $(K=3$; see Methods; Supplemental Appendix). To investigate the performance of the MCLE with changing parameters, we used Brent's search algorithm (see Methods) to estimate $\theta$ or $R$ with all other parameters fixed.

Black dots in Figure 1 show the ratios of 5\%, 25\%, 50\%, 75\%, and $95 \%$ percentiles of MCLE of $\theta[\operatorname{MCLE}(\theta)]$ versus the true value, with increasing $\varepsilon, n, \theta$, and $R$. Figure 2 is similar to Figure 1 , but shows the ratio of $\operatorname{MCLE}(R)$ versus $R$. The medians of $\operatorname{MCLE}(\theta)$ and $\operatorname{MCLE}(R)$ fit well with the true values, with exception when $\varepsilon$ is large or when $n$ is large (details given below). The distribution of $\operatorname{MCLE}(\theta)$ is more or less symmetric, while the distribution of $\operatorname{MCLE}(R)$ has a heavy right tail, which biases the mean of $\operatorname{MCLE}(R)$ upward. Increasing $R$ increases the variance of $\operatorname{MCLE}(\theta) / \theta$. The variance of $\operatorname{MCLE}(R) / R$ also increases with increasing $R$, except when $R$ is near 0 . In contrast, an increase of $n$ or $\theta / L$ decreases the variance of $\operatorname{MCLE}(\theta) / \theta$ and $\operatorname{MCLE}(R) / R$. With an increase of $\varepsilon$, we observe the variance of $\operatorname{MCLE}(R) / R$ increases with increasing $\varepsilon$ and then decreases a little bit when $\varepsilon$ is high (Fig. 2A). We also observe that the $\operatorname{MCLE}(\theta)$ is biased upward when $\varepsilon$ is large $\left(\varepsilon=10^{-3}\right.$, Fig. 1A) or when $n$ is large ( $n=2000,2500$, Fig. 1B). Both the observations are due to the fact that when $\varepsilon$ or $n$ is near or out of the maximum applicable range of the MCLEs, $\operatorname{MCLE}(R)$ tends to underestimate $R$ while $\operatorname{MCLE}(\theta)$ tends to overestimate $\theta$. The gray dots in Figures $1 \mathrm{~A}, \mathrm{~B}$ and $2 \mathrm{~A}, \mathrm{~B}$ are MCLEs calculated with assumption of, at most, four errors on any nucleotide site $(K=4)$. As we can see, by increasing the maximum allowable errors, we can obtain unbiased MCLEs.

\section{Compared to other $\theta$ estimators}

Assuming constant population size and unknown ancestral states of the nucleotide alleles, we used coalescent simulation to compare $\operatorname{MCLE}(\theta)$ with some other $\theta$ estimators that take into account error or are robust to error, i.e., two modified $\hat{\theta}_{S}$ estimators $\left(\hat{\theta}_{S c}\right.$ [Johnson and Slatkin 2008] and $\hat{\theta}_{S-\eta_{1}}$ [Achaz 2008]), two modified $\hat{\theta}_{\pi}$

\section{Genome Research}




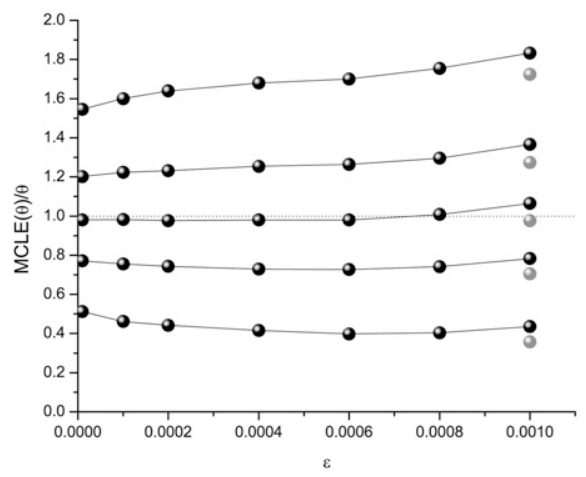

A
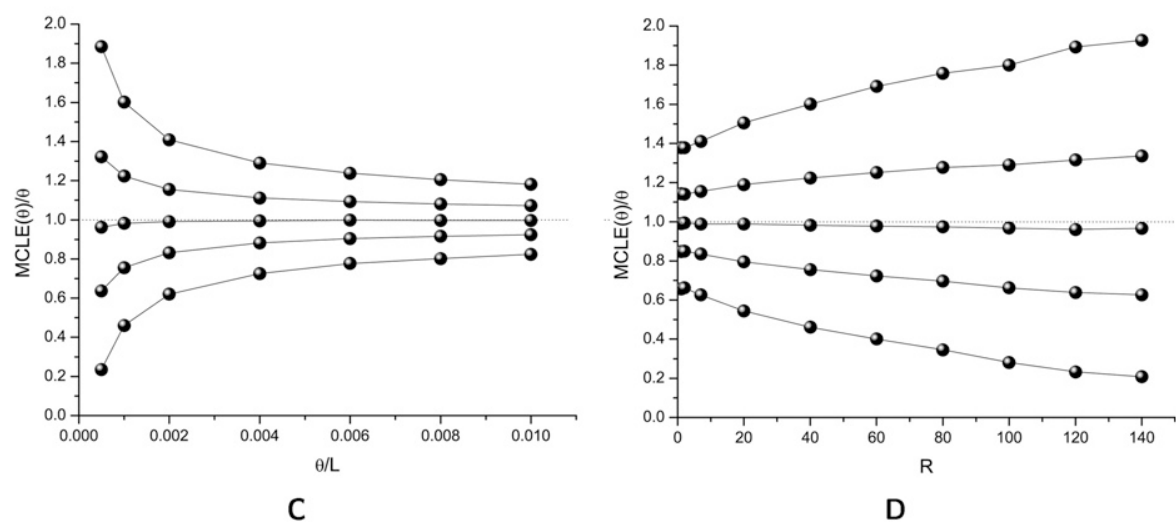

Figure 1. Effects of $\varepsilon(A), n(B), \theta / L(C)$, and $R(D)$ on the MCLE of $\theta$. The five point-and-lines (top to bottom) represent $95 \%, 75 \%, 50 \%, 25 \%$, and $5 \%$ percentiles of the MCLE, respectively. Unless shown on the $x$-axis, $\varepsilon=10^{-4}, n=200, \theta / L=10^{-3}, R=40$, and $L=10^{4}$. Black dots were percentiles of MCLEs calculated with $K=3$ and gray dots were percentiles of MCLEs calculated with $K=4$.

estimators $\left(\hat{\theta}_{\pi c}\right.$ [Johnson and Slatkin 2008] and $\hat{\theta}_{\pi-\eta_{1}}$ [Achaz 2008]), and two modified BLUE estimators ( $\hat{\theta}_{G L S f}$ and $\hat{\theta}_{B L U E-\eta_{1}}$ [Liu et al. 2009]). To provide performance standards, $\hat{\theta}_{S}$ and $\hat{\theta}_{\pi}$ were also calculated using the TRUE SNP spectrum (i.e., no errors), which were designated as $\hat{\theta}_{S_{\text {true }}}$ and $\hat{\theta}_{\pi_{\text {true }}}$, respectively (see Methods). Among those estimators, $\hat{\theta}_{S-\eta_{1}}$ and $\hat{\theta}_{\pi-\eta_{1}}$ assume an unknown error rate $\varepsilon$, while $\hat{\theta}_{S c}$ and $\hat{\theta}_{\pi c}$ assume known $\varepsilon$. Our $\operatorname{MCLE}(\theta)$ can be calculated with either known or unknown $\varepsilon$. In the former case, Brent's algorithm was used to find $\operatorname{MCLE}(\theta)$. In the latter case, we used Powell's algorithm (see Methods) to find the MCLE of $\theta$ and $\varepsilon$ simultaneously, but reported $\operatorname{MCLE}(\theta)$ only (treating $\varepsilon$ as a nuisance parameter). For each set of parameters, 10,000 samples were simulated and the mean, variance, and the root mean squared error (RMSE) of each estimator were calculated.

The results are shown in Figure $3 \operatorname{MCLE}(\theta)$ with either known or unknown $\varepsilon$ is the overall best estimator with all ranges of parameters tested. It is always among the group of the most accurate estimators in the comparison group as measured by the smallest RMSE. It shows clear advantage over other estimators with either high error rate or large sample size. $\operatorname{MCLE}(\theta)$ typically shows the same trend as $\hat{\theta}_{s_{\text {true }}}$ with the change of the parameters, but never

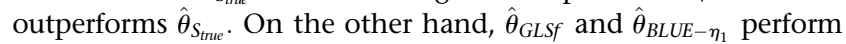
poorly with high error rates or large sample sizes, which is expected. The two modified $\hat{\theta}_{\pi}$ estimators have very similar performance as $\hat{\theta}_{\pi_{\text {true }}}$, but they never outperform $\operatorname{MCLE}(\theta)$ throughout the range of parameters tested, due to their large variances. The RMSEs of $\hat{\theta}_{S c}, \hat{\theta}_{G L S f}, \hat{\theta}_{B L U E-\eta_{1}}$, and $\hat{\theta}_{S-\eta_{1}}$ increase with increasing $\varepsilon$. For $\hat{\theta}_{S c}$, this is largely due to the increase of variance, while for the remaining three this is largely because they are biased upward with increasing $\varepsilon$. With increasing $n, \operatorname{MCLE}(\theta)$ becomes more accurate while $\hat{\theta}_{S c}$ becomes less accurate, which is due to increased variance. The RMSEs of $\hat{\theta}_{S-\eta_{1}}$, $\hat{\theta}_{G L S f}$, and $\hat{\theta}_{B L U E-\eta_{1}}$ first decrease and then increase with increasing $n$. The reason for $\hat{\theta}_{S-\eta_{1}}$ is that although its variance decreases with increasing $n$, it is biased upward at the same time. At a given range of relative small $n, \hat{\theta}_{G L S f}$ and $\hat{\theta}_{B L U E-\eta_{1}}$ are unbiased estimators and their variances decrease with increasing $n$. While $n$ is larger than the range, they are biased upward and their variances increase with increasing $n$. Both $\hat{\theta}_{\pi c}$ and $\hat{\theta}_{\pi-\eta_{1}}$ seem to be insensitive to $n$. With increasing $L$, all estimators become more accurate, with the only exception of $\hat{\theta}_{G L S f}$, which is biased upward with large $L$. On the other hand, while all estimators become more accurate with increasing $\theta / L, \hat{\theta}_{G L S f}$ outperforms all other estimators (even $\hat{\theta}_{S_{\text {true }}}$ ) with large $\theta / L$, due to its small variance. With increasing recombination rate $\rho$, all estimators increase their accuracy. At one extreme, when all nucleotide sites are independent, the RMSEs of $\operatorname{MCLE}(\theta)$ can go down to $0.144 \times \theta$, while that of $\hat{\theta}_{S_{\text {true }}}$ is $0.131 \times \theta$ (data not shown). Finally, we investigated the effect of the missing data rate $\delta$, which is defined as the number of nucleotide alleles excluded from analysis (due to low-quality read, PCR failure, among others) divided by the total number of alleles actually sequenced. As expected, $\operatorname{MCLE}(\theta)$ is relatively insensitive to $\delta$. However, it was unexpected to observe that with increasing $\delta$, only $\hat{\theta}_{S c}$ becomes less efficient, while $\hat{\theta}_{G L S f}$ actually becomes slightly more efficient. After a closer look, we believe that this is due to the fact that the missing data have two possibly positive effects on the estimators. First, it biases the $\theta$ estimators downward, which to some extent compensates for the upward bias caused by errors. Second, the higher the missing data rate, the higher the chance an error is called missing, which is similar to the effect of removing putative errors by increasing the standard of tolerable quality scores in sequence reading. Therefore, most of the estimators compared here should be able to be applied to data sets with some extent of missing data. Further investigation is needed to clarify their tolerable missing data rate.

\section{Applied to ANGPTL4 sequence data}

Romeo et al. (2007) showed a significant excess of rare alleles in the exon regions of the ANGPTL4 gene from 1832 African Americans and 1045 European Americans (for details, see Methods; Supplemental Fig. S1). There can be different explanations for this observation, including purifying selection, population expansion, and sequencing error. However, the three neutrality tests the authors used, Tajima (1989)'s $D$ and Fu and Li (1993)'s $D$ and $D^{*}$, cannot distinguish artificial significance (i.e., sequencing error) from biological significance (e.g., purifying selection and population expansion), because all assume no sequencing error in the data. 


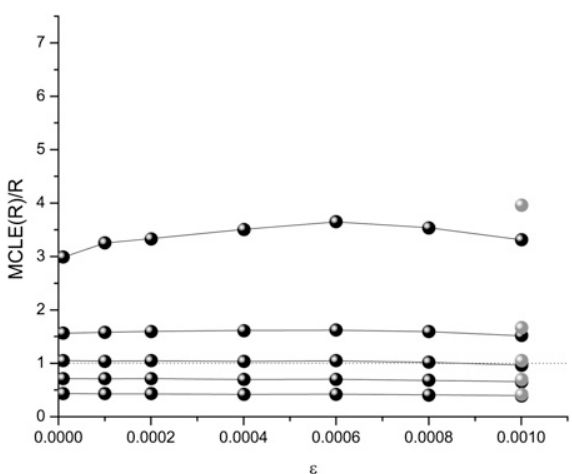

A

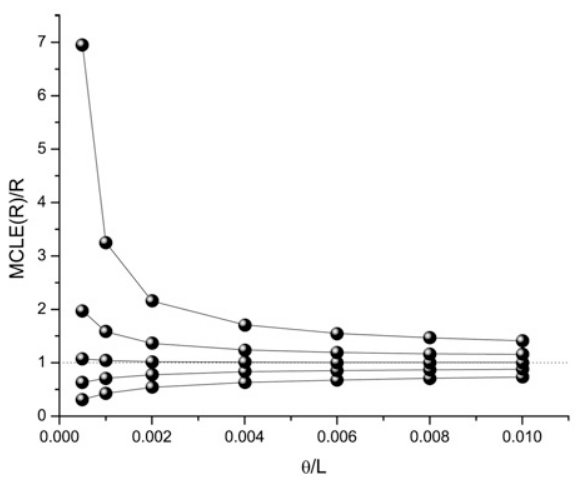

C

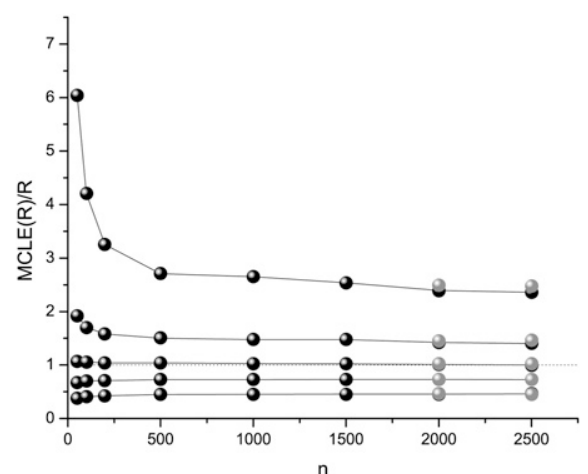

B

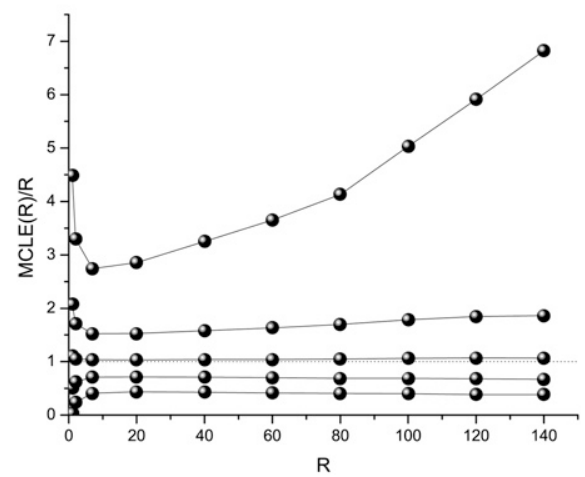

D

Figure 2. Effects of $\varepsilon(A), n(B), \theta / L(C)$, and $R(D)$ on the MCLE of $R$. The five point-and-lines (top to bottom) represent $95 \%, 75 \%, 50 \%, 25 \%$, and $5 \%$ percentiles of the MCLE, respectively. Unless shown on the $x$-axis, $\varepsilon=10^{-4}, n=200, \theta / L=10^{-3}, R=40$, and $L=10^{4}$. Black dots were percentiles of MCLEs calculated with $K=3$ and gray dots were percentiles of MCLEs calculated with $K=4$.

To show that the excess of rare alleles has biological significance, we need to test it with an error model. First, with an assumption of no error, we estimated $\operatorname{MCLE}(\theta)$ s as 0.0020 for European Americans and 0.0026 for African Americans, respectively. Then we compared the model without error (null hypothesis) to the model with error (alternative hypothesis) using parametric bootstrap with the $\operatorname{MCLE}(\theta)$ s (see Methods). The results showed that the alternative hypothesis is significantly better than the null hypothesis ( $P$-value smaller than 0.01 , one tail) based on 10,000 coalescent simulations, for both African Americans and European Americans. This suggests that sequencing error explains the excess of rare alleles better than the null hypothesis of no error.

Then we used a simple grid search method to estimate MCLEs of $\theta$ and $\varepsilon$ simultaneously (assuming constant population size), with step widths $10^{-4}$ and $10^{-6}$ for $\theta$ and $\varepsilon$, respectively. We assume there are at most three errors at any nucleotide site, which will guarantee an applicable $\operatorname{MCLE}(\theta)$ with an $\varepsilon$ up to $7.5 \times 10^{-5}$ (see Methods for explanation). For the African Americans, the estimated $\operatorname{MCLE}(\theta)$ is 0.0017 . Using parametric bootstrap, we estimated its approximate 95\% confidence interval (CI) (0.0010, $0.0027)$ based on 10,000 coalescent simulations. The estimated $\operatorname{MCLE}(\varepsilon)$ for African Americans is $3 \times 10^{-6}$. The same analysis was performed with European Americans. The results are $\operatorname{MCLE}(\theta)=$ 0.0011 , with 95\% CI $(0.0006,0.0018)$; $\operatorname{MCLE}(\varepsilon)=4 \times 10^{-6}$.

To test neutrality under the error model, we conducted coalescent simulation (10,000 replications) with the MCLEs of $\theta$ and $\varepsilon$, to obtain the null distributions of the test statistics. Based on the empirical null distributions, empirical $P$-values of one-tail tests for an excess of rare alleles (test statistics significantly smaller than expected) were obtained. Besides the above three test statistics we added Achaz (2008)'s $Y$ and $Y^{*}$ tests, which are modified Tajima (1989)'s $D$ and supposed to be more robust to errors. We also conducted a model goodness-of-fit test using the composite likelihood (see Methods) to test the overall model goodness-of-fit. The results of the tests are as follows. For African Americans, only Achaz's $Y(P=0.045)$ and $Y^{\star}(P=0.045)$ rejected the null hypothesis of neutrality. For the European Americans, Tajima's $D(P=0.044)$ and Achaz's $Y$ $(P=0.020)$ and $Y^{*}(P=0.020)$ rejected the null hypothesis. Fu and Li (1993)'s $D$ and $D^{*}$ and our model goodness-of-fit test failed to reject the null hypothesis in both cases. With the fact that the rare nonsynonymous changes have been verified experimentally (Romeo et al. 2007), singletons should have a lower error rate than other SNPs. Because this information is not incorporated in the current model, our MCLE $(\varepsilon)$ should be an upper limit of $\varepsilon$. Even with an error model that has overestimated $\varepsilon, Y$ and $Y^{\star}$ rejected the null hypothesis of no excess of rare alleles, which suggests that sequencing error cannot explain the excess of rare alleles. This leaves alternative explanations including purifying selection (Romeo et al. 2007), population expansion, or mixed effects of multiple acting factors as mentioned above to explain the excess. The results also suggested that Tajima's $D$ and Fu and Li's $D$ and $D^{*}$ tests have higher type I error rates when there are sequencing errors and lose power under the error model. Model goodness-of-fit tests using composite likelihood may be too conservative because of no specified alternative hypothesis. The neutrality tests that take error into account, such as Achaz's $Y$ and $Y^{*}$, should be preferred if there are likely many errors in the sequences.

Finally, assuming an exponential growth model, we used a hybrid algorithm to estimate MCLEs of $\theta, \varepsilon$, and $R$ simultaneously (searching $\theta$ and $\varepsilon$ using Powell's algorithm on a grid of $R$; see Methods for details). For African Americans, we estimated MCLE $(\theta)=$ $0.0027, \operatorname{MCLE}(\varepsilon)=2.3 \times 10^{-6}$ and $\operatorname{MCLE}(R)=5($ grid width $=1)$. With 10,000 parametric bootstrap samples, we estimated their approximate $95 \%$ CIs, which are $(0.0018,0.0040),\left(3 \times 10^{-7}, 6.8 \times\right.$ $\left.10^{-6}\right)$, and $(1.4,19.3)$, respectively. For European Americans, the corresponding estimates are: $\operatorname{MCLE}(\theta)=0.0121$ with $95 \% \mathrm{CI}$ $(0.0080,0.0166), \operatorname{MCLE}(\varepsilon)=1.7 \times 10^{-6}$ with $95 \%$ CI $(0,4.0 \times$ $\left.10^{-6}\right)$, and $\operatorname{MCLE}(R)=738$ with $95 \% \operatorname{CI}(404.2,1517.4)$.

\section{Discussion}

In this paper we propose a method based on the composite likelihood of SNP configurations for estimating population genetic parameters, such as the population mutation rate $\theta$ and scaled exponential growth rate $R$. There are several advantages of this method compared to available parameter estimation methods that incorporate error. First, it is relatively unbiased and can be

\section{Genome Research}




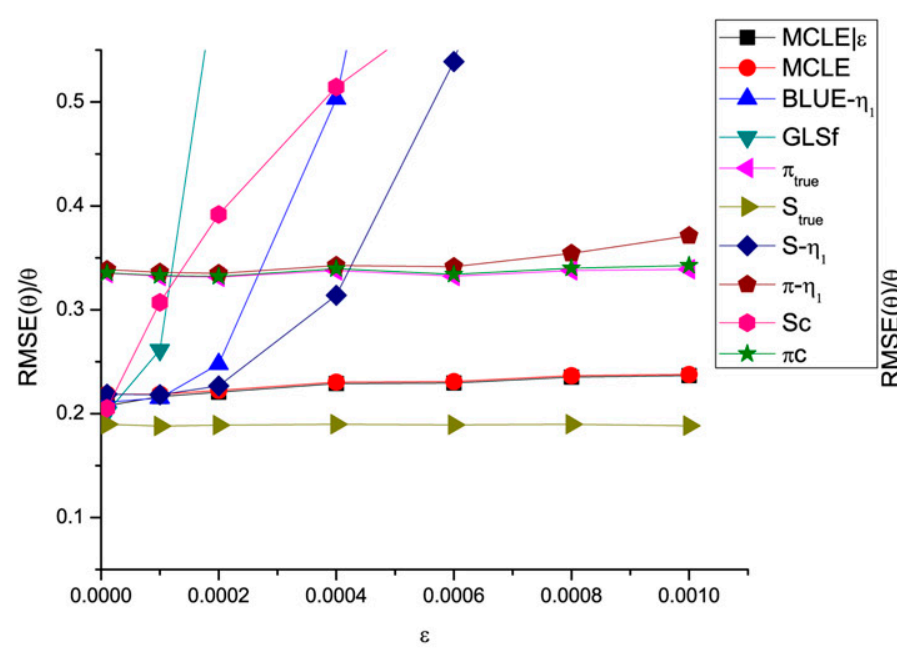

A
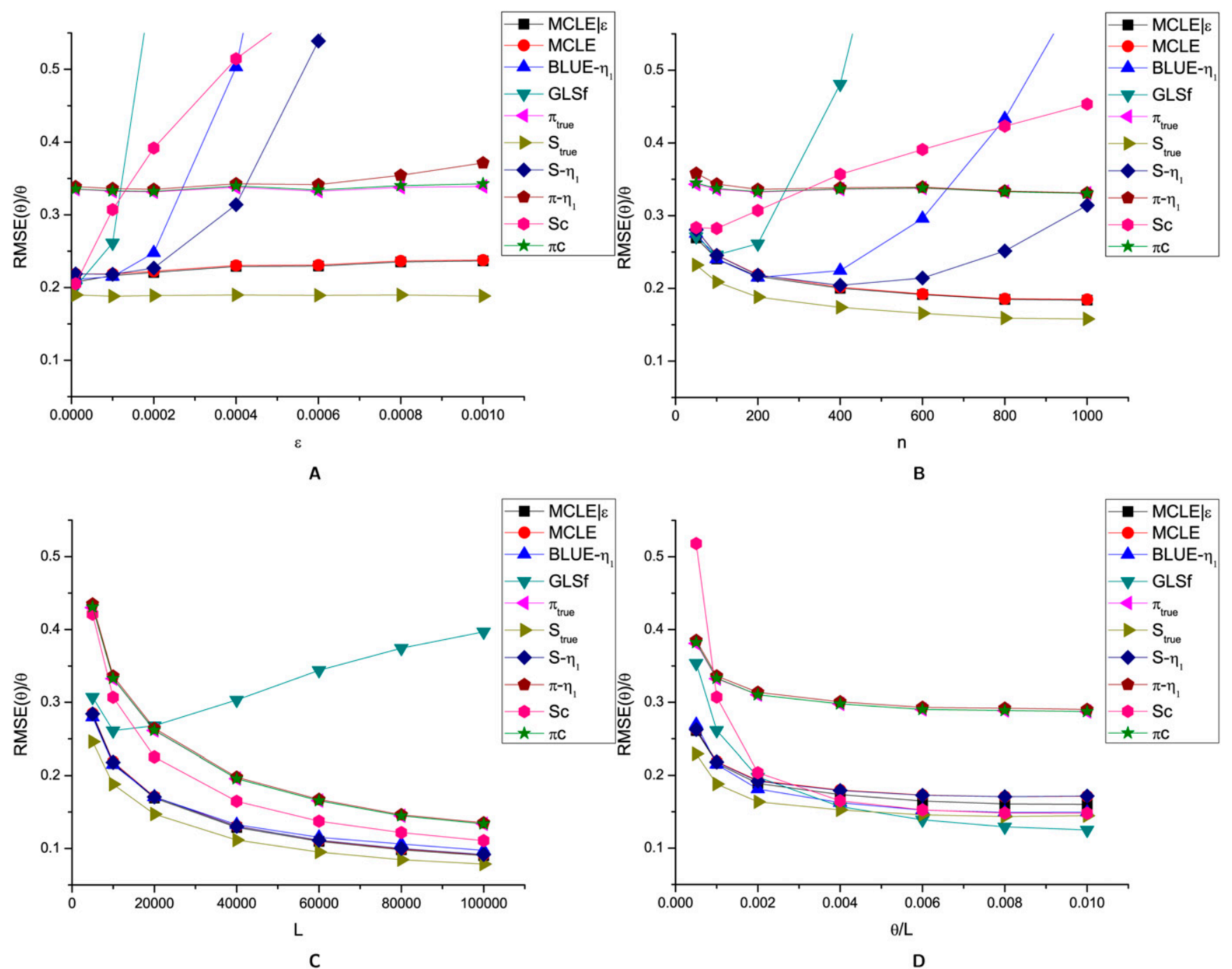

B

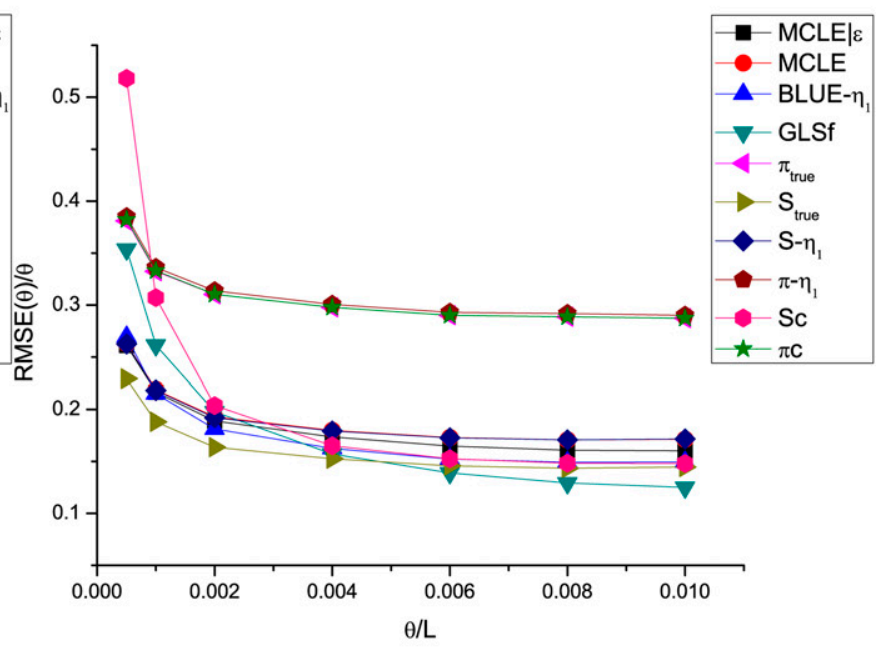

D
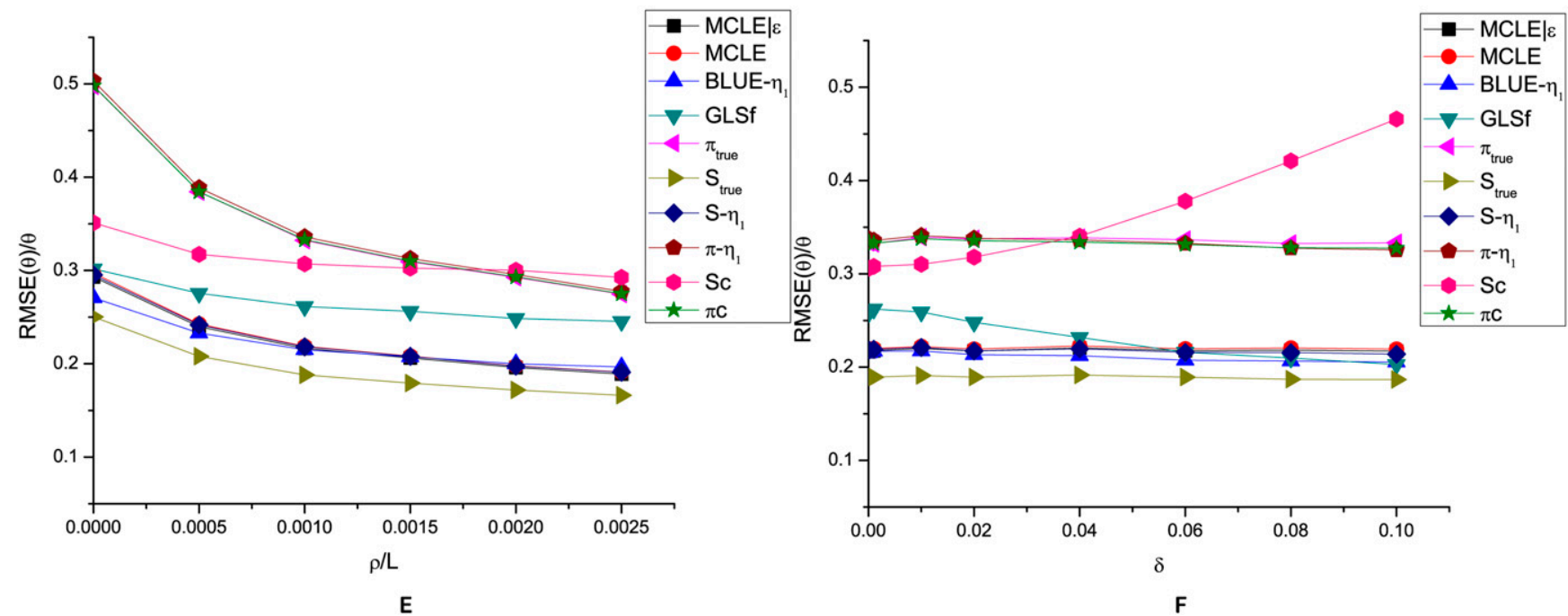

Figure 3. Effects of $\varepsilon(A), n(B), L(C), \theta / L(D), \rho / L(E)$, and $\delta(F)$ on the root mean squared error of different $\theta$ estimators. $\operatorname{MCLE} \mid \varepsilon$ : $\operatorname{MCLE}(\theta)$ assuming known $\varepsilon ; \operatorname{MCLE}: \operatorname{MCLE}(\theta)$ assuming unknown $\varepsilon ;$ BLUE- $\eta_{1}: \hat{\theta}_{B L U E-\eta_{1}} ;$ GLSf $: \hat{\theta}_{G L S f} ; \pi_{\text {true }}: \hat{\theta}_{\pi_{\text {ture }}} ; S_{\text {true }}: \hat{\theta}_{S_{\text {ture }}} ; S-\eta_{1}: \hat{\theta}_{S-\eta_{1}} ; \pi-\eta_{1}: \hat{\theta}_{\pi-\eta_{1}} ; S c: \hat{\theta}_{S c} ; \pi c: \hat{\theta}_{\pi c}$. Unless shown on the $x$-axis, $\varepsilon=10^{-4}, n=200, \theta / L=10^{-3}, L=10^{4}, \rho / L=10^{-3}$, and $\delta=0$. 
efficiently computed with relatively large error rate $\varepsilon$ or sample size $n$. Second, it is very flexible and can be used to estimate different sets of parameters assuming different models. The only requirement is the expected probabilities of SNP configurations under the model, which can be obtained either analytically or via computer simulation. Third, it fully models SNP configurations with more than two alleles. Fourth, the method can naturally handle missing data.

One limitation of our method is that it uses a single error rate $\varepsilon$ for all sites and all sequences, which is an acceptable treatment when quality score or probability call of genotypes are unavailable or only an average error rate is known. When the quality score of each genotype is available, we can improve our method by using a site-specific error rate $\varepsilon_{i}$ for site $i$ instead of an average $\varepsilon$. That is, we replace $\varepsilon$ with $\varepsilon_{i}$ in Equation 3 , see below, and $\varepsilon_{i}$ can be calculated using quality scores. This treatment gains computational efficiency, but loses power because it does not make full use of the quality score of each genotype. However, calculating all combinations of possible SNP configurations using a quality score (as proposed in Johnson and Slatkin 2006) may cause a large computational burden when the sample size is large. Further development is needed to compute composite likelihood efficiently with quality scores.

Another limitation of our method is associated with the nature of the composite likelihood. Since the composite likelihood is not the true likelihood, it does not have some of the important properties of the true likelihood. For example, a likelihood ratio test using the composite likelihood may be biased. This is the reason we proposed to use a computational intensive parametric bootstrap method to compare models. Some kind of normalization is needed before comparing composite likelihoods directly. More thorough studies are needed to investigate the statistical properties of the composite likelihood.

As shown in the Results, the MCLE with known $\varepsilon$ only slightly outperforms the MCLE with unknown $\varepsilon$. However, in regard to the computational speed, the former should be at least several-fold faster than the latter. As a simple case, if a grid-search algorithm is used and $\varepsilon$ is searched on $g$ grids, then the former should be approximately $g$ times faster than the latter. If efficient search algorithms, such as Brent's algorithm for one-dimensional space or Powell's algorithm for multidimensional space (see Methods) is used, the search speed is largely affected by the initial value of the parameter(s). For example, in one comparison with 1000 simulated data sets with the default parameters used in Figure 3 (true $\theta=$ $10^{-3} / \mathrm{bp}$ and $\varepsilon=10^{-4} / \mathrm{bp}$ ), the MCLE with known $\varepsilon$ (via Brent's algorithm with initial $\theta=2 \times 10^{-3} / \mathrm{bp}$ ) took $276 \mathrm{sec}$ to obtain the estimations; while the MCLE with unknown $\varepsilon$ (via Powell's algorithm with initial $\theta=2 \times 10^{-3} / \mathrm{bp}$ and $\varepsilon=10^{-5} / \mathrm{bp}$ ) took $1294 \mathrm{sec}$ on a PC using an Intel Pentium $4 \mathrm{CPU}$ at $3.4 \mathrm{GHz}$. Applied to the same data set, Brent's algorithm with initial $\theta=4 \times 10^{-3} / \mathrm{bp}$, took $286 \mathrm{sec}$ to obtain the estimations; while Powell's algorithm with initial $\theta=4 \times 10^{-3} / \mathrm{bp}$ and $\varepsilon=10^{-5} / \mathrm{bp}$ took $2217 \mathrm{sec}$.

As mentioned in the Introduction, several $\theta$ estimators, including ours, have been proposed to incorporate sequencing error or to be robust to errors. So far, extensive comparison of these estimators is limited. However, these estimators are designed for analyzing different types of data and all have their own advantages and disadvantages. Here we try to provide our recommendations for choosing the appropriate estimators based on our understanding and experience. First, when the sample size is small (tens of sequences), especially when the missing data rate is high, e.g., metagenomic sequencing data, Johnson and Slatkin (2006)'s
MCLE or Knudsen and Miyamoto (2007, 2009)'s full likelihood method is probably the best choice. The former is especially designed for metagenomic sequences when the quality score of each nucleotide read is available. The latter uses a model assuming no recombination. Therefore, if recombination is significant in the DNA sequences studied, Knudsen and Miyamoto (2007, 2009)'s method may not be appropriate. Second, when the sample size is large (thousands or even tens of thousands of sequences), the MCLE method proposed here is a suitable estimator to use. Third, when the sample size is moderate (hundreds of sequences), there are more choices. Johnson and Slatkin (2006)'s and our MCLEs may still be quite usable when the sample size is at the lower end or higher end of the hundreds, respectively. When the missing data rate and the error rate are relatively low, Liu et al. (2009)'s GLS estimator is a good choice. If the missing data rate is high or the error rate is high, Achaz (2008)'s corrected Watterson's estimator performs well with very little computational intensity.

One obvious dilemma in a resequencing study is how to balance sample size, the number of genes for sequencing and sequencing error. With a fixed budget, increasing sequencing coverage for each sample decreases the error rate, but at the same time decreases the total number samples or genes to be sequenced. A larger sample size provides higher power to discover rare alleles, but decreases the number of genes to be sequenced. At the same time, error accumulates linearly with sample size and makes it harder to identify true "signals" from "noises." Although answering this question is beyond the scope of this paper, our study does provide some clues. First, any methods using simple SNP counting regardless of errors, such as Watterson (1975)'s $\hat{\theta}_{S}$, may dramatically lose power when errors cannot be ignored (Liu et al. 2009). Second, using appropriate methods, such as our MCLE method, it is possible to achieve a similar power with higher error rates, which means that a smaller budget is needed for sequencing. Third, the power gain of methods using the SNP spectrum may diminish with increasing sample size, but the diminishing rate may be different for different estimators or different tests. For example, the variance of $\operatorname{MCLE}(\theta)$ decreases slower than $\operatorname{MCLE}(R)$ with increasing sample size. This means for different estimators or tests, the choice between more samples and fewer genes or more genes and smaller sample may be different.

In this study, we build a probability model for the SNP spectrum with $\theta, R$, and $\varepsilon$. With this model, we can estimate the three parameters by maximizing the composite likelihood of the spectrum. So far, we have been focused on estimating $\theta$ and $R$, but also demonstrate the estimation of $\varepsilon$ using the ANGPTL4 gene. Actually, with our limited comparison, MCLE of $\varepsilon$ shows a higher efficiency compared to the simple $\varepsilon$ estimations using $\hat{\theta}_{B L U E-\eta_{1}}$ and $\hat{\theta}_{S-\eta_{1}}$ (Liu et al. 2009; Supplemental Fig. S2). However, our model is not proper for other kinds of errors contained in the DNA sequences, including clone errors, PCR errors, and DNA degradation, all of which need specific probability models (e.g., Rambaut et al. 2009).

Several java programs for calculating MCLE of $\theta, R$, and $\varepsilon$, and core programs for calculating $\operatorname{MCLE}(\theta)$ are available at http:// sites.google.com/site/jpopgen/.

\section{Methods}

\section{Composite likelihood calculation}

Let us first define the configuration of a SNP as a (possibly partially ordered) serial of allele counting at that site, in which the first number is the counting of the ancestral alleles, while the second (and third, fourth, when they exist) is the counting of derived 
alleles. For example, at a site we observed 10 " $\mathrm{A}$ " alleles, five " $\mathrm{C}$ " alleles, and one " $\mathrm{T}$ " allele, and we know the " $\mathrm{A}$ " alleles are the ancestral alleles. Then the configuration of that site is presented as $[10,5,1]$ or $[10,1,5]$. That is, the numbers in [ ] are partially ordered, so that $[10,5,1] \neq[5,10,1]$, but $[10,5,1]=[10,1,5]$. On the other hand, if the ancestral state is unknown, we present the configuration as a set of unordered counting of alleles. In the previous example, if we do not know which allele is ancestral, then we represent its configuration as $\{10,5,1\}$, or $\{5,10,1\}$, or $\{1$, $5,10\}$, etc.

We designate $\Gamma_{i}^{T}$ as the true allele configuration of site $i$, with the ancestral state either known or unknown. Assuming the infinite sites model, there are at most two alleles at a given site. Let us separate $\Gamma_{i}^{T}$ for two different conditions, ancestral state known or unknown. Suppose at site $i$ there are $j$ ancestral alleles and $n_{i}-j$ mutant alleles, where $n_{i}$ is the number of observed alleles on that site. If we assume a constant population size, the expected probability of observing a true configuration $\left[j, n_{i}-j\right]$ is

$$
\operatorname{Pr}\left(\left[j, n_{i}-j\right] \mid \dot{\theta}\right)=\left\{\begin{array}{ll}
\dot{\theta} /\left(n_{i}-j\right) & \text { if } 1 \leq j \leq n_{i}-1 \\
1-a_{n_{i}} \dot{\theta} & \text { if } j=n_{i}
\end{array},\right.
$$

where $\operatorname{Pr}()$ means probability, $a_{n}=1+\frac{1}{2}+\cdots+\frac{1}{n-1}$, and $\dot{\theta}$ is $\theta$ per base pair $(\dot{\theta} \ll 1)$ (Fu 1995). In an exponential growth model for the population, $N_{e}(t)=N_{e}(0) \exp (-r t)$, where $N_{e}(t)$ is the effective population size $t$ generations before the current time (generation 0 ). In population genetics, a scaled population growth rate, $R=2 N_{e} r$ for diploids or $R=N_{e} r$ for haploids, is often used as a demographic parameter. The expected probability of observing a true configuration $\left[j, n_{i}-j\right], \operatorname{Pr}\left(\left[j, n_{i}-j\right] \mid \dot{\theta}, R\right)$ can be calculated using formulas described in Polanski et al. (2003) and Polanski and Kimmel (2003). More generally, assuming any demographic models and/or mutation models, $\operatorname{Pr}\left(\left[j, n_{i}-j\right] \mid \dot{\theta}, \Omega\right)$ can be calculated either analytically, numerically or using a Monte Carlo simulation, given a set of model parameters $\Omega$, other than $\dot{\theta}$. If $\operatorname{Pr}\left(\left[j, n_{i}-j\right] \mid \dot{\theta}, \Omega\right)$ has to be evaluated via simulation and $n_{i}$ is different from site to site because of missing data, then it will be more efficient to evaluate only $\operatorname{Pr}\left(\left[k, n_{\max }-k\right] \mid \dot{\theta}\right)$ using simulation, where $n_{\max }$ is the maximum of all $n_{i}$, and calculate

$$
\begin{aligned}
\operatorname{Pr}\left(\left[j, n_{i}-j\right] \mid \dot{\theta}\right)= & \frac{1}{\left(\begin{array}{c}
n_{\max } \\
n_{i}
\end{array}\right)} \sum_{k=j}^{n_{\max }-n_{i}+j} \operatorname{Pr}\left(\left[k, n_{\max }-k\right] \mid \dot{\theta}\right)\left(\begin{array}{l}
k \\
j
\end{array}\right) \\
& \times\left(\begin{array}{c}
n_{\max }-k \\
n_{i}-j
\end{array}\right)
\end{aligned}
$$

for $n_{i} \neq n_{\text {max }}$.

If the ancestral state of the site is unknown, then

$$
\begin{aligned}
\operatorname{Pr} & \left(\left\{j, n_{i}-j\right\} \mid \dot{\theta}, \Omega\right) \\
\quad & \begin{cases}\operatorname{Pr}\left(\left[j, n_{i}-j\right] \mid \dot{\theta}, \Omega\right)+\operatorname{Pr}\left(\left[n_{i}-j, j\right] \mid \dot{\theta}, \Omega\right) & \text { if } 1 \leq \min \left(j, n_{i}-j\right)<n_{i} / 2 \\
\operatorname{Pr}\left(\left[j, n_{i}-j\right] \mid \dot{\theta}, \Omega\right) & \text { if } j=n_{i} / 2 \\
1-\sum_{k=1}^{n_{i}-1} \operatorname{Pr}\left(\left[k, n_{i}-k\right] \mid \dot{\theta}, \Omega\right) & \text { if } \min \left(j, n_{i}-j\right)=0,\end{cases}
\end{aligned}
$$

where $\min ()$ means minimum.

We designate $\Gamma_{i}^{O}$ as the observed allele configuration of site $i$, with ancestral state either known or unknown. $\Gamma_{i}^{O}$ can be different from $\Gamma_{i}^{T}$ because of sequencing error. Given the error rate $\varepsilon$ and $\Gamma_{i}^{T}$, we can calculate the expected probability $\operatorname{Pr}\left(\Gamma_{i}^{O} \mid \Gamma_{i}^{T}, \varepsilon\right)$ (details given below). Then

$$
\operatorname{Pr}\left(\Gamma_{i}^{O} \mid \dot{\theta}, \varepsilon, \Omega\right)=\sum_{\Gamma_{i}^{T}} \operatorname{Pr}\left(\Gamma_{i}^{O} \mid \Gamma_{i}^{T}, \varepsilon\right) \operatorname{Pr}\left(\Gamma_{i}^{T} \mid \dot{\theta}, \Omega\right) .
$$

Then a composite likelihood (CL) of a range of sequence is calculated as the product of the expected probability of the observed allele configuration of each site. That is,

$$
C L=\prod_{i} \operatorname{Pr}\left(\Gamma_{i}^{O} \mid \dot{\theta}, \varepsilon, \Omega\right) .
$$

\section{Probability of observed SNP configuration}

Here we explain how to calculate $\operatorname{Pr}\left(\Gamma_{i}^{O} \mid \Gamma_{i}^{T}, \varepsilon\right)$. Let us assume that on a given site the true SNP configuration is either $[i, j]$ or $\{i, j\}$. Actually we do not need to distinguish $[i, j]$ or $\{i, j\}$ in this step because they are treated as the same. So in the following we simply use counting of the different allele types to represent the observed configuration of SNP, in which there may be more than two types of alleles because of error. For example, $(i+1, j-2,1)$ represents an observed SNP configuration with one type of allele with $i+1$ counts, another type of allele with $j-2$ counts, and a third allele with one count.

First, we assume there are, at most, $K$ errors on any site. The choosing of $K$ depends on $L, n$, and $\varepsilon$. A $K$ that is too small may violate the assumption that there are at most $K$ errors at any site, therefore the estimation will be biased. However, a larger $K$ may significantly increase the computational burden. As a rule of thumb, we can choose the $K$ as the minimum integer satisfying $L \times$ $[1-$ binomCDF $(n, K, \varepsilon)]<0.5$, where binomCDF is the binomial cumulative distribution function that returns the probability of sequencing error, which occurs with a probability $\varepsilon$, occurring $K$ or less times in $n$ trials. However, $\varepsilon$ is unknown in practice, therefore we choose a $K$ to guarantee an applicable estimation with an upper limit of $\varepsilon$. For example, the largest $n$ in the ANGPTL4 data equals $1832 \times 2=3664$ and $L=2604$. Therefore, $K=2$ will guarantee an applicable estimation with an $\varepsilon$ up to $2.9 \times 10^{-5}$, while $K=3$ corresponds to an $\varepsilon$ up to $7.5 \times 10^{-5}$.

Now we show how to calculate the probabilities of different observed SNP configurations given $i, j$, and $\varepsilon$, assuming $\Gamma_{i}^{T}$ is either $[i, j]$ or $\{i, j\}$. Without loss of generality let us assume the allele with $i$ copies is " $\mathrm{A}$ " and the other allele with $j$ copies is "C." Let us further assume that there are at most $m(m \geq 2)$ possible types of alleles at a site (if only point mutations are considered then $m=4$ ), and when a sequencing error occurs at an allele, the allele has an equal probability $u=1 /(m-1)$ of changing to another type of allele. For the following, we show the derivation of the probabilities assuming there are at most two errors on each site $(K=2)$.

1. We consider three different cases, that is, there are 0,1 , or 2 sequencing errors on the site:

(1) There is 0 sequencing error:

Following a binomial distribution, this event has probability

$$
\operatorname{Pr}(i, j)=(1-\varepsilon)^{i+j} .
$$

(2) There is 1 sequencing error:

If the error occurs at an " $\mathrm{A}$ " allele, the event can be further divided into two different cases. In the first case the error changes the "A" allele to a " $\mathrm{C}$ " allele, which produces a configuration $(i-1, j+1)$ with probability

$$
\operatorname{Pr}(i-1, j+1)=i u \varepsilon(1-\varepsilon)^{i+j-1} .
$$

In the second case the error changes the " $\mathrm{A}$ " allele to a " $\mathrm{G}$ " or " $\mathrm{T}$ " allele, which produces a configuration $(i-1, j, 1)$ with probability

$$
\operatorname{Pr}(i-1, j, 1)=i(1-u) \varepsilon(1-\varepsilon)^{i+j-1} .
$$

Similarly, if the error occurs on a " $\mathrm{C}$ " allele, the event produces the configuration $(i+1, j-1)$ or $(i, j-1,1)$. We can easily calculate 
$\operatorname{Pr}(i+1, j-1)$ and $\operatorname{Pr}(i, j-1,1)$ by switching $i$ and $j$ in Equations 5 and 6.

(3) There are 2 sequencing errors:

There are a total of 13 different possible configurations that can be produced by two errors. Their probabilities can be calculated as

$$
\begin{aligned}
\operatorname{Pr}(i, j) & =i j u^{2} \varepsilon^{2}(1-\varepsilon)^{i+j-2} \\
\operatorname{Pr}(i-1, j, 1) & =i j u(1-u) \varepsilon^{2}(1-\varepsilon)^{i+j-2} \\
\operatorname{Pr}(i-1, j-1,2) & =i j u(1-u) \varepsilon^{2}(1-\varepsilon)^{i+j-2} \\
\operatorname{Pr}(i-1, j-1,1,1) & =i(1-u)(1-2 u) \varepsilon^{2}(1-\varepsilon)^{i+j-2} \\
\operatorname{Pr}(i-2, j+2) & =\left(\begin{array}{c}
i \\
2
\end{array}\right) u^{2} \varepsilon^{2}(1-\varepsilon)^{i+j-2} \\
\operatorname{Pr}(i-2, j+1,1) & =\left(\begin{array}{c}
i \\
1
\end{array}\right)\left(\begin{array}{c}
i-1 \\
1
\end{array}\right) u(1-u) \varepsilon^{2}(1-\varepsilon)^{i+j-2} \\
\operatorname{Pr}(i-2, j, 2) & =\left(\begin{array}{c}
i \\
2
\end{array}\right) u(1-u) \varepsilon^{2}(1-\varepsilon)^{i+j-2} \\
\operatorname{Pr}(i-2, j, 1,1) & =\left(\begin{array}{c}
i \\
2
\end{array}\right)(1-u)(1-2 u) \varepsilon^{2}(1-\varepsilon)^{i+j-2} .
\end{aligned}
$$

By switching $i$ and $j$, we can obtain $\operatorname{Pr}(i, j-1,1), \operatorname{Pr}(i+2, j-2), \operatorname{Pr}(i+$ $1, j-2,1), \operatorname{Pr}(i, j-2,2)$, and $\operatorname{Pr}(i, j-2,1,1)$.

2. We combine the probabilities that produce the same configuration. Specifically,

$$
\begin{aligned}
\operatorname{Pr}(i, j) & =(1-\varepsilon)^{i+j}+i j u^{2} \varepsilon^{2}(1-\varepsilon)^{i+j-2} \\
\operatorname{Pr}(i-1, j, 1) & =i(1-u) \varepsilon(1-\varepsilon)^{i+j-1}+i j u(1-u) \varepsilon^{2}(1-\varepsilon)^{i+j-2} \\
\operatorname{Pr}(i, j-1,1) & =j(1-u) \varepsilon(1-\varepsilon)^{i+j-1}+i j u(1-u) \varepsilon^{2}(1-\varepsilon)^{i+j-2} .
\end{aligned}
$$

There are 32 possible configurations when there are three errors at a site. Their probabilities and combined probabilities for $K=3$ and a method to compute the probabilities with $K \geq 4$ can be found in the Supplemental Appendix.

\section{Parameter estimation}

Given the composite likelihood function (Equation 4), we can estimate the parameters based on the criterion of maximum composite likelihood. The simplest method is a grid search. Other algorithms are available for efficiently searching the parameter space for the optimal estimate without derivatives, such as Brent's algorithm for a one-dimensional space and Powell's algorithm or the downhill simplex algorithm for a multidimensional space (Press et al. 2007). Our limited experience shows that Brent's algorithm works reasonably well in most cases (estimating either $\theta$ or $R$ ). When estimating $\theta$ and $\varepsilon$ simultaneously, both Powell's algorithm and the downhill simplex algorithm work reasonably well, while Powell's algorithm slightly outperforms the downhill simplex algorithm. On the contrary, when estimating $\theta$ and $R$ simultaneously, the downhill simplex algorithm slightly outperforms Powell's algorithm, but it often fails to find the global maximum composite likelihood estimate. If all three parameters $(\theta, R$, and $\varepsilon)$ need to be estimated, both Powell's algorithm and the downhill simplex algorithm perform badly. In that case, grid search is a slow but reliable choice. A faster alternative is some kind of hybrid search algorithm, e.g., using Powell's algorithm to search the MCLEs of $\theta$ and $\varepsilon$ on a grid of $R$.

Parametric bootstrap can be used to estimate an approximate confidence interval of a MCLE of a parameter. After the MCLEs of the parameters of a given model are estimated using the original data (designated as $\overline{M C L E}$ ), coalescent simulation is conducted with the $\overline{M C L E}$. With each replication, a sample of sequences with the same sample size and the same length as the original data are simulated. Then the same missing data pattern on each site of the original data is superimposed onto the simulated sequence sample. Finally, using the simulated sample, the MCLE of the given parameter (designated as $\overline{M C L E}$ ) is estimated with all other parameters fixed to their $\overline{M C L E}$. After a large number of replications, the $\overline{M C L E}$ s of the given parameter form an empirical distribution from which the confidence interval of the $\widehat{M C L E}$ of the parameter can be approximated.

\section{Model goodness-of-fit test and model comparison}

To test the model goodness-of-fit, we first calculate the MCLE of all parameters of the model, $\widehat{M C L E}$. Then a parametric bootstrap is conducted as described above. For each simulated sample, the set of MCLE(s) of the parameters for the model, $\overline{M C L E}$, is estimated, with a corresponding composite likelihood, $\widetilde{C L}$. The resulting composite likelihoods of all simulated samples (designated as $\widetilde{C L}{ }_{n}$ ) is considered as an empirical null distribution of the composite likelihood assuming $\overline{M C L E}$ is the true model parameter. Finally, the composite likelihood of the observed sample (designated as $C L_{o}$ ) is compared to the empirical null distribution. Given a small fraction $\alpha$, if $C L_{o}$ is smaller than $\alpha / 2$ or larger than $1-\alpha / 2$ of all $\widetilde{C L}{ }_{n}$ 's, we reject the null hypothesis of the model at the $\alpha$ level (twotail test). Since recombination reduces the variance of the composite likelihood, a simulation of sequences without recombination will make the test more conservative.

Since composite likelihood is not full likelihood, a simple likelihood ratio test cannot be directly applied. Fortunately, parametric bootstrap can also be used for simple model comparisons. For example, suppose we want to compare two different neutral population models with constant population size. The null model assumes $\varepsilon=0$ and has one parameter $\theta$. The alternative model makes no such assumption and has two parameters, $\theta$ and $\varepsilon$. To perform the comparison, we first estimate the MCLE(s) of the two models, designated as $\widehat{M C L E}_{1}$ and $\widehat{M C L E}_{2}$ for the null and the alternative models, respectively. The corresponding CL's are designated as $C L_{1}$ and $C L_{2}$, respectively. Parametric bootstrap is conducted with the $\overline{M C L E}_{1}$. For each simulated sample, the set of MCLE(s) of the parameters for the null model (in this case only $\theta$ ), $\widetilde{M C L E}_{1}$, is estimated, with a corresponding composite likelihood, $\widetilde{C L}_{1}$. With the same simulated sample, the set of MCLEs of the parameters for the alternative model (in this case, $\theta$ and $\varepsilon$ ), $\overline{M C L E}_{2}$, is estimated with a corresponding composite likelihood $\widetilde{C L} L_{2}$. Then the difference of the two composite likelihoods $\Delta=\widetilde{C L}_{2}-\widetilde{C L}_{1}$ is calculated. With all replications, $\Delta$ forms an empirical null distribution with an assumption of the null model. Given a small fraction $\alpha$, if $C L_{2}-C L_{1}$ is larger than $1-\alpha$ of the null distribution, we conclude that the alternative model is significantly better than the first model at the $\alpha$ level (one tail).

\section{Computer simulation}

Coalescent simulations (e.g., Hudson 2002) were used to validate our MCLE and compare the performance of different $\theta$ estimators. We assumed a Wright-Fisher model, neutrality, and the infinite sites model for mutation and unknown ancestral allele state. When validating the MCLE, we simulated sequences in an exponential growth population with parameter $R$ and without recombination. When comparing the MCLE with other $\theta$ estimators, we simulated sequences with a given recombination rate $\rho$ in a neutral population with constant size. When conducting model goodness-offit tests and model comparison for the ANGPTL4 gene, we simulated sequences assuming a neutral population with constant size and without recombination. Sequencing error on each site was

\section{Genome Research}


simulated to follow a binomial distribution with parameter $n \varepsilon$. Only point mutations $(m=4)$ were simulated. Missing data were simulated by random removal of simulated alleles according to the missing data rate.

\section{Other $\theta$ estimators compared}

As mentioned in the Introduction, there are several new $\theta$ estimators that incorporate error or are supposed to be robust to error. We selected six estimators that can be efficiently calculated with a relatively large sample size to be compared with our MCLE of $\theta$. We calculated the estimators assuming that the ancestral states of the alleles are unknown. All estimators assume constant population size and neutrality. As standards of performance, two widely used $\theta$ estimators, Tajima (1983)'s estimator based on the average difference between two sequences $(\pi)$ and Watterson (1975)'s estimator based on the total number of polymorphic sites $(S)$, were also calculated with the simulated TRUE allele frequencies (i.e., no errors). Those two estimators were designated as $\hat{\theta}_{\pi_{\text {true }}}$ and $\hat{\theta}_{s_{\text {true }}}$, respectively.

Two of the estimators we selected for comparison are modified Tajima (1983)'s estimators. They are Johnson and Slatkin (2008)'s $\hat{\theta}_{\pi c}$, assuming known $\varepsilon$, and Achaz (2008)'s $\hat{\theta}_{\pi-\eta_{1}}$, assuming unknown $\varepsilon$. Another two estimators selected are modified Watterson (1975)'s estimators: Johnson and Slatkin (2008)'s $\hat{\theta}_{S c}$, assuming known $\varepsilon$, and Achaz (2008)'s $\hat{\theta}_{S-\eta_{1}}$, assuming unknown $\varepsilon$. The remaining two estimators for comparison were recently proposed by Liu et al. (2009). They are the modified Fu (1994)'s BLUE estimators, assuming either known $\varepsilon$ (designated as $\hat{\theta}_{G L S f}$ ) or unknown $\varepsilon\left(\hat{\theta}_{B L U E-\eta_{1}}\right)$. Because those two estimators cannot handle missing data directly, when there are alleles missing on a nucleotide site we imputed them according to the observed allele frequency of that site. Detailed description of the calculation of the above six estimators can be found in Liu et al. (2009).

Johnson and Slatkin (2006)'s estimator was not compared because of the requirement of a quality score for each nucleotide allele. Lynch $(2008,2009)$ 's method based on $\pi$ was not compared because of the requirement of sequence coverage information for each individual. However, with high coverage available, Lynch $(2008,2009)$ 's estimation is supposed to be close to $\hat{\theta}_{\pi_{\text {true }}}$.

\section{ANGPTL4 sequence data}

The sequence data set of the ANGPTL4 gene was from the Dallas Heart Study (see Romeo et al. 2007, 2009 for a detailed description of the data and their supplemental tables for the SNP spectrum we analyzed in this study). The sequencing region consists of seven exons and the intron-exon boundaries of the gene, with a total length of $2604 \mathrm{bp}$. The randomly sampled individuals include 1832 African Americans, 1045 European Americans, 601 Hispanic, and 75 other ethnicities. In this study we only analyzed African American and European American data. There are a total of 79 polymorphic sites (excluding insertion/deletions) combining African Americans and European Americans (60 in African Americans alone and 44 European Americans alone). Because the missing data information is unavailable for the monomorphic sites, we used an average missing data rate of $6.24 \%$, which is estimated from the polymorphic sites.

\section{Acknowledgments}

This research was supported by the MiCorTex study and National Institutes of Health grant 5P50GM065509. We thank Jonathan C. Cohen for kindly providing the ANGPTL4 data. We thank the three anonymous reviewers for their comments and suggestions. We thank Sara Barton for her help with polishing the language.

\section{References}

Achaz G. 2008. Testing for neutrality in samples with sequencing errors. Genetics 179: 1409-1424.

Clark AG, Whittam TS. 1992. Sequencing errors and molecular evolutionary analysis. Mol Biol Evol 9: 744-752.

$\mathrm{Fu}$ YX. 1994. Estimating effective population size or mutation rate using the frequencies of mutations of various classes in a sample of DNA sequences. Genetics 138: 1375-1386.

Fu YX. 1995. Statistical properties of segregating sites. Theor Popul Biol 48: 172-197.

Fu YX, Li WH. 1993. Statistical tests of neutrality of mutations. Genetics 133: 693-709.

Hellmann I, Mang Y, Gu Z, Li P, de la Vega FM, Clark AG, Nielsen R. 2008. Population genetic analysis of shotgun assemblies of genomic sequences from multiple individuals. Genome Res 18: 1020-1029.

Hudson RR. 2001. Two-locus sampling distributions and their application. Genetics 159: 1805-1817.

Hudson RR. 2002. Generating samples under a Wright-Fisher neutral model of genetic variation. Bioinformatics 18: 337-338.

Jiang R, Tavaré S, Marjoram P. 2009. Population genetic inference from resequencing data. Genetics 181: 187-197.

Johnson PLF, Slatkin M. 2006. Inference of population genetic parameters in metagenomics: A clean look at messy data. Genome Res 16: 1320-1327.

Johnson PLF, Slatkin M. 2008. Accounting for bias from sequencing error in population genetic estimates. Mol Biol Evol 25: 199-206.

Knudsen B, Miyamoto MM. 2007. Incorporating experimental design and error into coalescent/mutation models of population history. Genetics 176: $2335-2342$.

Knudsen B, Miyamoto M. 2009. Accurate and fast methods to estimate the population mutation rate from error prone sequences. $B M C$ Bioinformatics 10: 247. doi: 10.1186/1471-2105-10-247.

Liu X, Maxwell TJ, Boerwinkle E, Fu Y-X. 2009. Inferring population mutation rate and sequencing error rate using the SNP frequency spectrum in a sample of DNA sequences. Mol Biol Evol 26: 1479-1490.

Lynch M. 2008. Estimation of nucleotide diversity, disequilibrium coefficients, and mutation rates from high-coverage genomesequencing projects. Mol Biol Evol 25: 2409-2419.

Lynch M. 2009. Estimation of allele frequencies from high-coverage genome-sequencing projects. Genetics 182: 295-301.

Nielsen R. 2000. Estimation of population parameters and recombination rates from single nucleotide polymorphisms. Genetics 154: 931-942.

Polanski A, Kimmel M. 2003. New explicit expressions for relative frequencies of single-nucleotide polymorphisms with application to statistical inference on population growth. Genetics 165: 427-436.

Polanski A, Bobrowski A, Kimmel M. 2003. A note on distributions of times to coalescence, under time-dependent population size. Theor Popul Biol 63: 33-40.

Press WH, Teukolsky SA, Vetterling WT, Flannery BP. 2007: Numerical recipes: The art of scientific computing, 3rd ed. Cambridge University Press.

Rambaut A, Ho SYW, Drummond AJ, Shapiro B. 2009. Accommodating the effect of ancient dna damage on inferences of demographic histories. Mol Biol Evol 26: 245-248.

Romeo S, Pennacchio LA, Fu Y, Boerwinkle E, Tybjaerg-Hansen A, Hobbs $\mathrm{HH}$, Cohen JC. 2007. Population-based resequencing of ANGPTL4 uncovers variations that reduce triglycerides and increase HDL. Nat Genet 39: 513-516.

Romeo S, Yin W, Kozlitina J, Pennacchio LA, Boerwinkle E, Hobbs HH, Cohen JC. 2009. Rare loss-of-function mutations in angptl family members contribute to plasma triglyceride levels in humans. J Clin Invest 119: 70-79.

Shendure J, Ji H. 2008. Next-generation DNA sequencing. Nat Biotechnol 26: 1135-1145.

Tajima F. 1983. Evolutionary relationship of DNA-sequences in finite populations. Genetics 105: 437-460.

Tajima F. 1989. Statistical method for testing the neutral mutation hypothesis by DNA polymorphism. Genetics 123: $585-595$.

Watterson GA. 1975. Number of segregating sites in genetic models without recombination. Theor Popul Biol 7: 256-276.

Whitaker RJ, Banfield JF. 2006. Population genomics in natural microbial communities. Trends Ecol Evol 21: 508-516.

Zwick ME. 2005. A genome sequencing center in every lab. Eur J Hum Genet 13: $1167-1168$.

Received June 18, 2009; accepted in revised form October 8, 2009. 


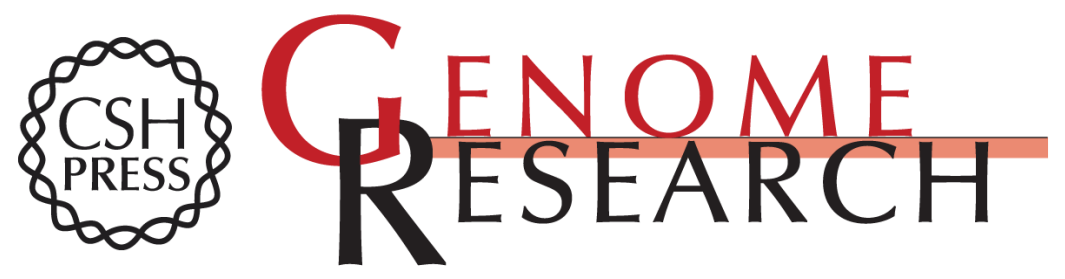

\section{Estimating population genetic parameters and comparing model goodness-of-fit using DNA sequences with error}

Xiaoming Liu, Yun-Xin Fu, Taylor J. Maxwell, et al.

Genome Res. 2010 20: 101-109 originally published online December 1, 2009

Access the most recent version at doi:10.1101/gr.097543.109

Supplemental http://genome.cshlp.org/content/suppl/2009/11/05/gr.097543.109.DC1

Material

References This article cites 28 articles, 2 of which can be accessed free at: http://genome.cshlp.org/content/20/1/101.full.html\#ref-list-1

Open Access Freely available online through the Genome Research Open Access option.

License Freely available online through the Genome Research Open Access option.

Email Alerting Receive free email alerts when new articles cite this article - sign up in the box at the Service top right corner of the article or click here.

\section{Affordable, Accurate Sequencing.}

